\title{
Apicoplast Biosynthetic Pathways as Possible Targets for Combination Therapy of Malaria
}

\author{
Solomon Tesfaye, Bhanu Prakash and Prati Pal Singh \\ Center of Infectious Diseases, Department of Pharmacology and Toxicology, National Institute of Pharmaceutical Education and \\ Research, India
}

\begin{abstract}
The emergence of malaria parasite strains resistant to practically all the antimalarial drugs in clinical use is now making it necessary to discover and develop both new antimalarial drugs and treatments. Recent advances in molecular techniques along with the availability of genome sequence of Plasmodium falciparum may provide a wide range of novel targets in metabolic pathways like isoprenoid biosynthesis, fatty acid biosynthesis and heme biosynthesis in the apicoplast of Plasmodium. On the other hand, the combination therapy approach (currently used to retard the selection of parasite strains resistant to individual components of a combination of drugs) has proved to be a success in the combination of sulphadoxine and pyrimethamine, which targets two different steps in the folate pathway of malaria parasite. However, after the success of this therapeutic combination, the efficacy of other combinations of drugs which target different enzymes in a particular metabolic pathway has, apparently, not been reported. Therefore, herein, we review various drug targets so far discovered in apicoplast-related anabolic pathways, especially, with a sharper focus on the possibility to target more than one enzyme at a time in a particular metabolic pathway of malaria parasites.
\end{abstract}

Key words: Apicoplast, combination therapy, fatty acid, isoprenoid, malaria, Plasmodium falciparum.

\section{Introduction}

In the era of huge concern on the life style diseases, even in the nations where a considerable number of population are suffering from old infectious diseases, malaria continues to be one of the leading causes of morbidity and mortality in the tropical and subtropical regions of the world. According to global malaria estimates, in the year 2013, nearly 3.2 billion people were at the risk, and 198 million clinical cases and 584,000 deaths occurred. Further, between 2000 and 2013, global expanded malaria interventions resulted in nearly $30 \%$ reduction in incidence and, malaria mortality rates were reduced by an estimated $47 \%$ worldwide [1]. The largest human population at the risk of malaria is in Africa, South-East Asia and western Pacific regions with the largest share going to Africa [2]. Moreover, the interaction between malaria and HIV has further aggravated the problem [3, 4].

Corresponding author: Prati Pal Singh, Ph.D., professor, research field: microbial and parasite chemotherapy: E-mail: drppsingh2005@gmail.com.
Other than health-related problems, malaria also incurs enormous economic burden both at national and household levels [5]. The current challenge in the control and treatment of malaria is the emergence, spread and consolidation of parasite strains (especially $P$. falciparum) resistant to conventional antimalarial drugs such as chloroquine and sulphadoxine-pyrimethamine [6]. Moreover, recently a decline in the effectiveness of artemesinin and its derivatives has been reported on Cambodia borders [7, 8]. Artemisnin-resistant $P$. falciparum strains, as monitored by the high prevalence of parasites carrying K 13 propeller mutations, are now rampant across Mayanmar and next to northeastern border of India [9]. The fear is that, if artemesinin-resistant $P$. falciparum strains are transmitted to the rest of the world, ACT (artemesinin-combination therapy), which has so far been the most effective first-line treatment option in many countries [10], may also meet the same fate as the other drugs. Therefore, the discovery and development of new, novel and affordable 
antimalarial drugs and of new treatments is an urgent necessicity.

Apicoplast, which is derived from an engulfed red alga, is present in most of apicomplexan parasites like Plasmodium [11]. Due to its evolutionary distance from the host, apicoplast is now considered as an important organelle for the targeted drug discovery [12-14]. Apicoplast houses prokaryotic machinery, presumably to replicate its circular $35-\mathrm{kb}$ genome and to transcribe and translate the genes that it possesses [15]. Treatment of malaria parasites with antibiotics such as ciprofloxacin, an inhibitor of the bacterial DNA gyrase, and translation blocking antibiotics including azithromycine, chloramphenicol, clindamycin, tetracycline and rifampicin results in the so called "delayed death" effect. During antibiotic treatment, which is not recommended for acute malaria [16], the arrest of cell growth occurs in the next asexual cycle, whereas the parasites in the current cycle, apparently remained relatively unaffected $[17$, 15, 18]. In addition to the study of housekeeping processes, apicoplast also offers opportunities to study various metabolic machineries such as isoprenoid, fatty acid and heme biosynthetic pathways alongwith their respective enzymes. Surprisingly, unlike the "delayed death" effect which has been observed as a consequence of the action(s) of some antibiotics, to target enzymes involved in these anabolic pathways resulted in defaying delayed death effect in $P$. falciparum [15]. Moreover, the known absence of some of the enzymes which constitute these pathways in eukaryotic hosts, makes them even more attractive, novel and ideal targets [19]. That is why, currently, investigations of the metabolic processes which occur in apicoplast constitute new prospects for new antimalarial drug discovery [20].

The concept of multipoint inhibition approach, which targets different enzymes in the same metabolic pathway, has been evidenced to be a success of different drug combinations which inhibit enzymes DHPS (dihydropteroate synthetase) and DHFR (dihydrofolate reductase) [21], and cystein and aspartic proteases [22]. Nevertheless, despite the clinical success of these drug combinations, and in the face of the fact that potential apicoplastic antimalarial drug targets/enzymes are evolutionary far distant from various putative targets in the human metabolome, no other drug combination(s) has been reported, which targets different enzymes in one particular anabolic pathway of apicoplast of malaria parasite. In this review, we discuss various drug targets discovered so far in the apicoplast anabolic pathways, with special focus on the possibilities of combining novel inhibitors or drugs to target them.

\section{Isoprenoid Biosynthesis Pathway}

Isoprenoid biosynthesis is one of the pathways in malaria parasite which partakes enzymes which are found in more than one compartment to produce its final products, isoprenoids. Isoprenoids are a diverse range of compounds which are assembled from two common five-carbon precursors known as IPP (isopentenyl pyrophosphate) and DMAPP (dimethyl-allyl diphosphate). Some of the known isoprenoids are (a) steroids which are involved in membrane structure, (b) heme $\mathrm{A}$ and ubiquinone which are believed to have significant contribution in electron transport, (c) dolichol that is required for glycoprotein synthesis, and (d) isopentyladenine that is present in some transfer RNAs [23]. The biosynthesis of isoprenoids depends on the condensation of different numbers of IPP units which are synthesized via two different biosynthetic routes viz. the mevalonate and non-mevalonate pathways [24]. In mammals, cytoplasm of plants, fungi, some bacteria and several protozoa, isoprenic units are derived from the typical mevalonate pathway [23]. In plastids of plants, several algae, eubacteria, cyanobacteria and apicomplexa, the MEP (methyl erythritol phosphate) pathway or DOXP (1-deoxy-D-xylulose 5-phosphate) pathway produces IPP and DMAPP [25]. 
Mevalonate pathway is an important metabolic pathway that provides cells with essential bioactive molecules, which are vital for multiple cellular processes. Additionally, this metabolic pathway converts mevalonate into sterol isoprenoids, such as cholesterol, lipoproteins, steroid hormones and several intermediate molecules [26]. These intermediates play important roles in the post-translational modification of a multitude of proteins involved in intracellular signaling, cell growth/differentiation, gene expression, apoptosis, meiosis, protein glycosylation and cytoskeletal assembly [27, 28]. This pathway depends on the condensation of three molecules of acetyl CoA (coenzyme A) into HMG-CoA (3-hydroxy-3-methylglutaryl coenzyme A), which is reduced to mevalonate by HMGCoA reductase. Mevalonate is further converted into IPP with mevalonate 5-phosphate as an intermediate. An important difference between cytoplasmic acetate/mevalonate pathway and the plastid DOXP pathway is that the former uses starting compounds of two-carbon building blocks, whereas the latter uses both two-carbon and three-carbon building blocks [28].

In apicoplast, triose phosphate importers and the subsequent modifying enzymes generate pyruvate along with glyceraldehydes-3-phosphate (GA3P) to be used by the first enzyme of the DOXP pathway, DOXP synthase (DXS; Fig. 1). DXS condenses pyruvate and GA3P to DOXP [29]; DOXP reductoisomerase (DXR) then catalyzes the intramolecular rearrangement and reduction of DOXP to MEP. Subsequent reactions which incorporate different enzymes convert MEP to IPP and DMAPP, which are used as intermediates in the production of isoprenoids such as dolichol and ubiquinones.

DOXP pathway, in addition to its hypothesized role in the formation of isopentenylated tRNAs inside apicoplast, also gives IPP and DMAPP as substrates for the downstream production of isoprenoids in cytoplasm. After their shipment to cytoplasm by poorly understood mechanisms, IPP and DMAPP are then condensed to GPP (geranyl diphosphate) by FPPS (farnesyl diphosphate synthase) [30]. GPP then condenses with the second IPP molecule to form FPP (farnesyl diphosphate). Finally, FPP is converted to GGPP (geranylgeranyl diphosphate) by GGPPS (geranylgeranyl diphosphate synthase, Fig. 1).

One of the drugs that targets the isoprenoid pathway is the antibiotic fosmidomycin (Fig. 2) that was isolated from Streptomyces lavendulae as an antibacterial agent in 1970's [31]. In contrast to other antibiotics which act on housekeeping processes located in apicoplast and cause the classic "delayed death" effect to the malaria parasite, fosmidomycin and its analogs cause a rapid parasite and fever clearance mainly due to the immediate ceassation of isoprenoid supply required for a variety of cellular functions of malaria parasite [32]. Initially, fosmidomycin was reported as a potent inhibitor of the DXR enzyme that is essential in the non-mevalonate pathway, which eventually blocks the biosynthesis of isopentenyl diphosphate and the subsequent formation of isoprenoids in $P$. falciparum [20]. However, recently, a second target of fosmidomycin, MCT (methylerythritol phosphate cytidyltransferase) has been reported [33]. The attempts of these authors to validate the biological effects of fosmidomycin in both P. falciparum and Escherchia coli using mass spectroscopy have shown the inhibition of the growth of both the organisms at the level of MCT.

A metabolite of known herbicide clomazone, 5-ketoclomazone, was also reported to block another enzyme DXS in the same biosynthesis pathway [34]. DXS, a thiamin pyrophosphate-dependent enzyme, was cloned in E. coli and the inhibition assay was done using radiolabelled pyruvate. Further, the authors made it clear that the parent compound (clomazone) has no effect on the activity of this enzyme; rather its metabolite (5-ketoclomazone) inhibited recombinant DXS of Chlamydomonas with an 


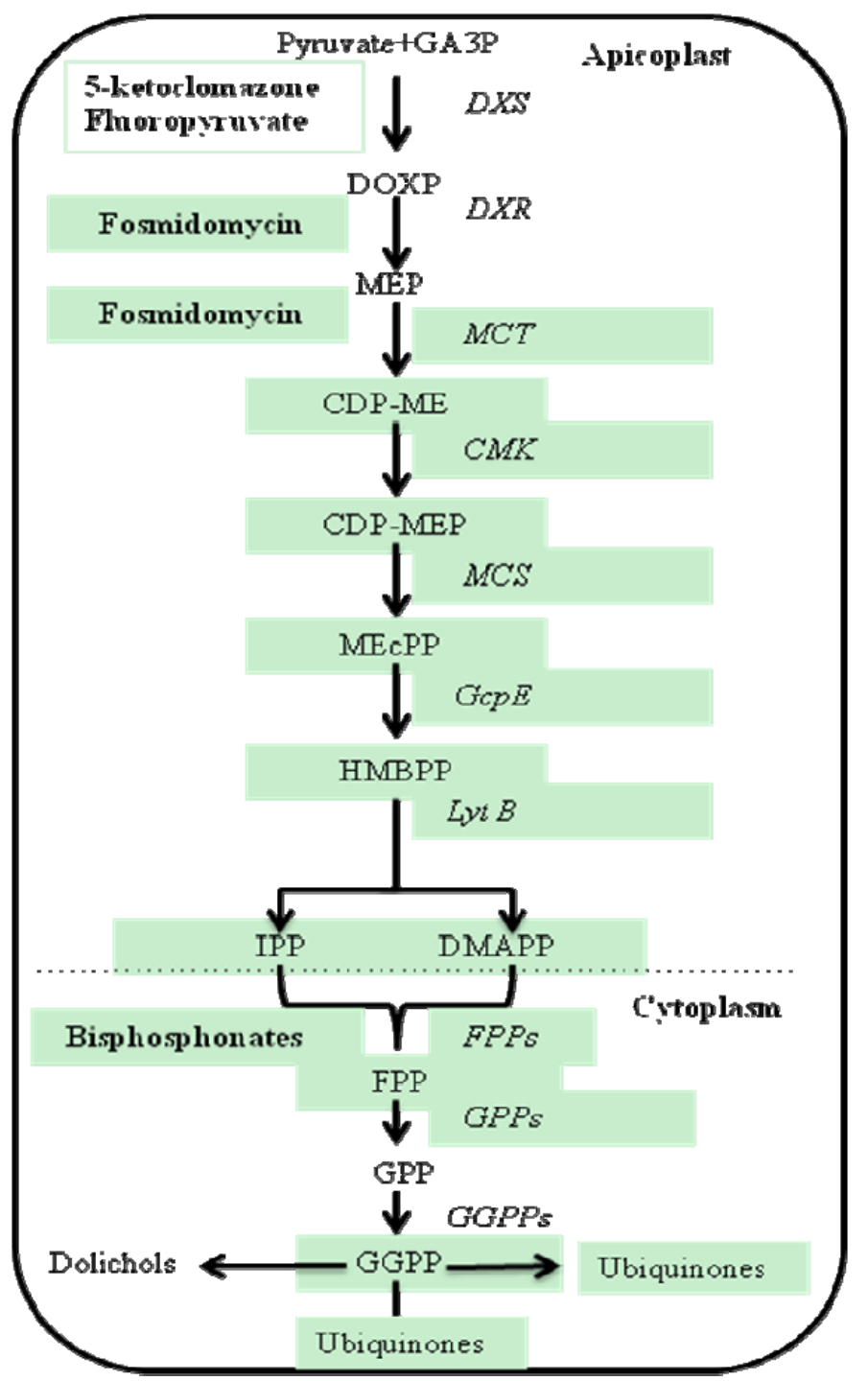

Fig. 1 The DOXP (above) and the cytoplasmic (below) parts of isoprenoid biosynthesis pathway in $P$. falciparum. 5-ketoclomazone, fosmidomycin and bisphosphonates inhibit DOXP synthase (DXS), [DOXP reductoisomarase (DXR) and methylerythritol phosphate cytidyl- transferase (MCT)] and farnesol diphosphate synthase (FPPs), respectively. GA3P, glyceraldehydes-3-phosphate; CDP-ME, 4-diphosphocytidyl-2-C-methyl-D-erythritol; $\quad$ CDP-MEP, 4-diphosphocytidyl-2-C-methyl-D-erythritol-2-phosphate; MEcPP, 2-C-methyl-D-erythritol-2,4 cyclodiphosphate; HMBPP, (E)-4-hydroxy-3-methyl-but-2-enyl diphosphate; IPP, isopentenyl diphosphate; FPPs, farnesyl diphosphate synthase; GPP, geranyldiphosphate; GPPs, geranyldiphosphate synthase; FPP, farensyl diphosphate; GGPP, geranylgeranyl diphosphate; GGPPs, geranylgeranyl diphosphate synthase.

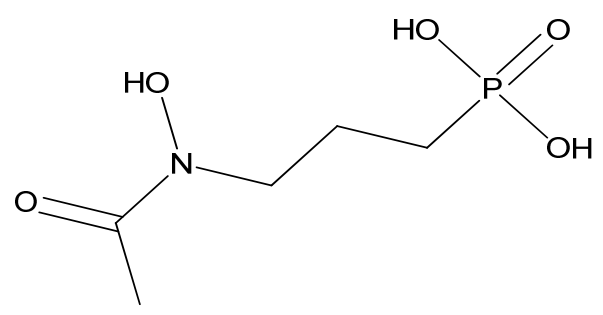

Fosmidomycin<smiles>CC(C)(P(=O)([O-])[O-])P(=O)([O-])O</smiles>

Bisphosphonates

Fig. 2 Inhibitors of isoprenoid biosynthesis pathway, general structure of fosmidomycin and bisphosphonates $\left(\mathbf{R}_{1}\right.$ and $\mathbf{R}_{2}$ indicate the positions of variable side chains). 
approximate $\mathrm{IC}_{50}$ value of $0.1 \mathrm{mM}$. Similarly, in another study, spectrophotometric assays showed that fluoropyruvate also inhibited DXS enzyme from Pseudomonas aeruginosa and E. coli with $\mathrm{IC}_{50}$ values of $400 \mu \mathrm{M}$ and $80 \mu \mathrm{M}$, respectively [35]. Fluoropyruvate is believed to bind covalently to the active site of DOXP synthase [28].

The last enzyme of DOXP pathway, Lyt B, has been reported to be involved in the catalysis of $\mathrm{PiSigma}(\pi / \sigma)$ metallacycle intermediates [36]. These authors showed the inhibition of Lyt B by a potent inhibitor, propargyl diphosphate, and suggested that formation of matallacycle complex (between the alkyne group of the compound and the fourth $\mathrm{Fe}$ of the $\mathrm{Fe}_{4} \mathrm{SO}_{4}$ complex of the enzyme) as a possible mechanism for inhibition. This particular work, which was done by using ENDOR (electron-nuclear double resonance) spectroscopy, gave an insight for the development of new inhibitors. Bisphosphonates is another group of drugs which acts on isoprenoid biosynthesis pathway (Fig. 2). They are pyrophosphate analogs in which the oxygen bridge between the two phosphorus atoms has been replaced by a carbon substituted with various side chains. These compounds, because of their inhibitory activity towards FPPS of osteoclast cells in bone, are clinically used as anti-boneresorptive drugs. Current studies regarding this group of drugs have reported that lipophilic bisphosphonates have better efficacy in the inhibition of FPPS as compared to the commercially available polar bisphosphonates [30, 37]. In contrast to the above mentioned studies, Jordao et al. (2011) [38] have reported that risedronate (hydrophilic bisphosphonate) had an $\mathrm{IC}_{50}$ of $20.3 \pm 1.0 \mu \mathrm{M}$ against $P$. falciparum, in vitro, and caused $88.9 \%$ inhibition of malaria parasite $P$. berghei in a rodent model, on the seventh day of treatment. Bisphosphonates also known to stimulate human $\gamma \delta \mathrm{T}$ cells [39] and, thus, may elicit antiparasitic immune response. Moreover, the inhibition of FPPS also results in the accumulation of the substrate IPP, which conjugates to AMP to form a toxic ATP analogue known as O-isopentenyl-ATP (ApppI) which inhibits the mitochondrial adenine nucleotide translocase and induces apoptosis [40].

Several independent works have reported the activities of several drugs, stand-alone, on various enzymes of non-mevalonate isoprenoid biosynthesis. However, our literature search, using PubMed search engine for published articles from 1996 onwards, on the combination of these drugs to target more than one enzyme at the same time, did not result in much evidence in this regard except only one study on $E$. coli [41]. This particular study reported the synergestic effect of a combination of fosmidomycin and bisphosphonates in the inhibition of the growth of E. coli K12. The enzymes which were targeted in $E$. coli isoprenoid biosynthesis pathway were similar to those in $P$. falciparum. However, apparently, there are no reports concerning the screening of these drugs for their synergistic activity against malaria parasites.

\section{Fatty Acid Biosynthesis Pathway}

Lipids, which are highly abundant components of all organisms, serve as building blocks of membranes, depots for energy storage and post-translational modifications which regulate the localization and function of proteins. Lipids have also been identified as pathogenetic factors for various infectious diseases [42]. Fatty acid biosynthesis is fundamental to cell growth, differentiation as well as homeostasis. Almost all living organisms including apicomplexan parasites such as $P$. falciparum synthesize fatty acids. Before the discovery and characterization of several $P$. falciparum FAS (fatty-acid synthesis) enzymes, it was believed that $P$. falciparum lacks a de novo FAS pathway [43]. However, this belief was disproved based on the results of the genomic sequencing of $P$. falciparum. The results confirmed that $P$. falciparum is capable of synthesizing its own fatty acids. Further, all the enzymes of FAS pathway are known to be present in apicoplast [44]. 
FAS pathway in apicomplexan parasites is different from that of their eukaryotic hosts in a number of aspects. For instance, unlike their eukaryotic hosts, FAS pathway of apicomplexan parasites is catalyzed by discrete enzymes, and is known as type II pathway or "dissociative" pathway [43]. On the other hand, mammalian FAS pathway (type I or associative pathway) takes place in cytosol, and all steps of this pathway are catalyzed by fatty acid synthase, a single giant polypeptide enzyme [16].

FAS pathway is one of the best studied pathways in apicoplast. This pathway is the main source for de novo synthesis of fatty acids and lipoic acid [42]. Lipoic acid is synthesized in both mitochondria and apicoplast by lipoic acid synthase enzyme. This acid is a potent antioxidant that may have an important role in protecting the parasite against oxidative stress during erythrocytic stage of its life cycle [45]. The main carbon source for this pathway is acetyl-CoA, which can either be generated from acetate through the action of acetyl-CoA synthetase or from pyruvate by PDHC (pyruvate dehydrogenase complex) [43]. In malaria parasite, apicoplast-localized PDHC is thought to be the primary source of acetyl-CoA [46]. The first enzyme in the pathway, ACCase (acetyl CoA carboxylase), converts acetyl-CoA to malonyl-CoA which is later changed to malonyl-acetyl carrier protein (malonyl-ACP) by malonyl-CoA: ACP transcylase (MCAT) [46] (Fig. 3). $\beta$-ketoaceyl-ACP synthase III catalyzes the condensation of malonyl-ACP with other acetyl-CoA to form a 3-oxoacyl-ACP product [47]. In the elongation process, OAR (3-oxoacyl-ACP reductase), after removing carbonyl group, reduces 3-oxoacyl-ACP to 3-hydroxyacyl-ACP that enters in dehydration cycle [48]. Once produced, 3-hydroxyacyl-ACP is converted into enoyl-ACP by $\beta$-hydroxy-acyl-ACP dehydratase and enters into the second reductive step (catalyzed by enoyl-ACP reductase) to complete the first round of elongation cycle (Fig. 3).

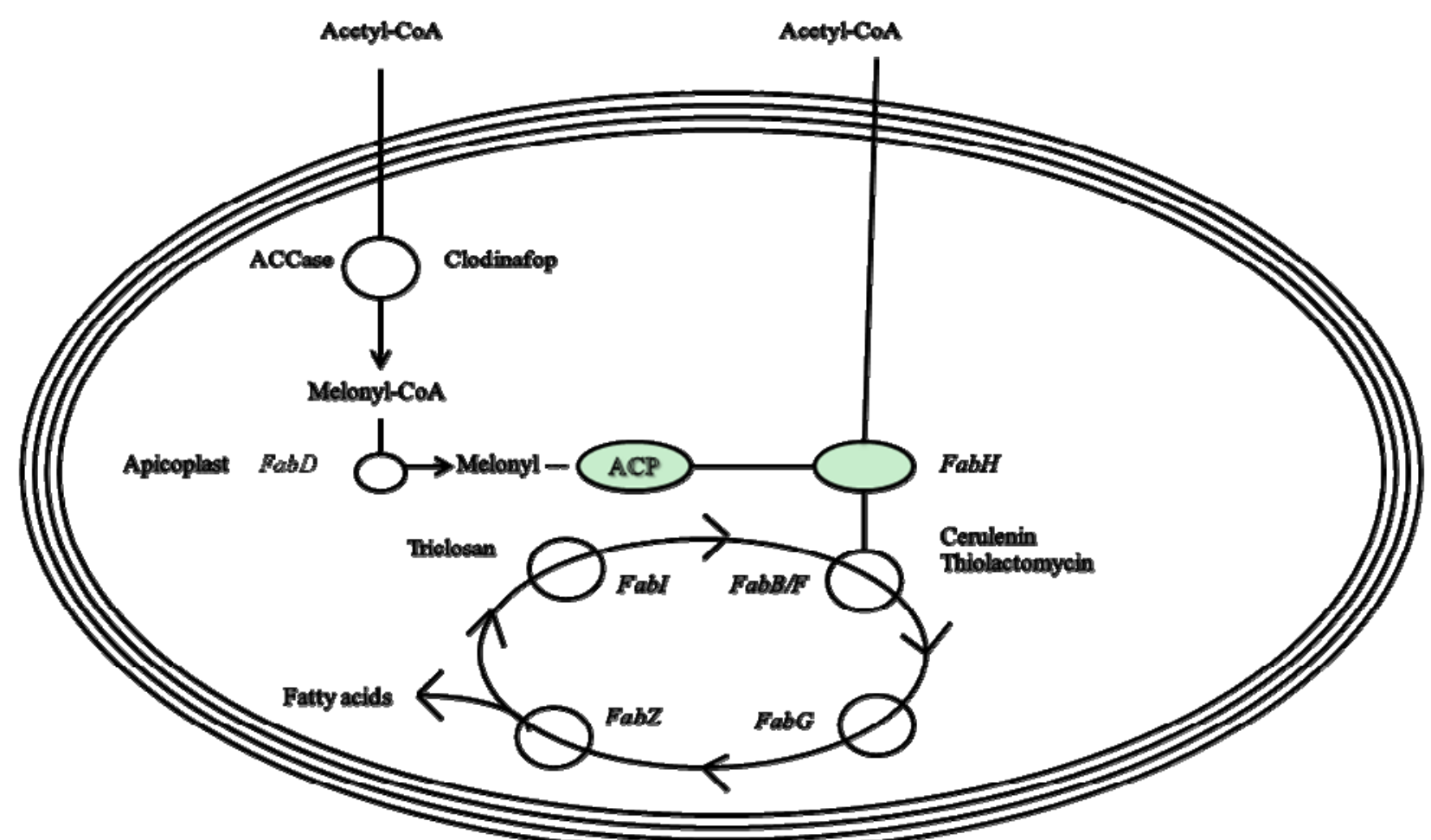

Fig. 3 Fatty acid synthesis II in apicoplast of P. falciparum. ACC, acetyl-CoA carboxylase; ACP, acyl carrier protein; FabD, ACP transacylase; FabH, $\beta$-ketoacyl-ACP synthase III; FabG, $\beta$-ketoacyl-ACP reductase; FabA, $\beta$-hydroxydecanoyl-ACP dehydratase/isomerase; FabZ, $\beta$-hydroxyacyl-ACP dehydatase; FabI, enoyl- ACP reductase; FabB, $\beta$-ketoacyl-ACP synthase I; FabF, $\beta$-ketoacyl-ACP synthase II. 
Several studies on FAS II have reported the inhibition of the growth of the blood-stage of Plasmodium by blocking various enzymes $[49,50,16]$. However, recent gene deletion experiments have proved that apicoplastic FAS II is only essential in the late liver stage of the life cycle of malaria parasite [51]. It has been demonstrated that the deletion of two critical enzymes of FAS II, Fab B/F and Fab Z, did not affect asexual blood stage replication [52]. Rather, the late liver stage of these parasites was unable to form exo-erythrocytic merozoites. In agreement with the above results, hexachlorophene (Fig. 4), a known inhibitor of Fab G [53], was accounted to inhibit liver stage growth in vitro [54]. Even more, it was reported that the growth of Fab I deficient P. falciparum in its blood-stage was not affected [51].

Despite the expression of FAS II enzyme genes, both in blood and mosquito stages and the upregulation of genes which encode type II FAS<smiles>Oc1c(Cl)cc(Cl)c(Cl)c1Cc1c(Cl)c(Cl)cc(Cl)c1Cl</smiles>

Hexachlorophene<smiles>C/C=C/C=C/CCCC(=O)[C@@H]1O[C@@H]1C(N)=O</smiles>

Ceruline enzymes in $P$. falciparum samples collected from patients in Senegal [55], recent reports have suggested that fatty acid biosynthesis is critical only for the late liver stage development of malaria parasite. Taking into account the importance of evaluating the reported inhibitors for pre-erythrocytic activity and checking their specific binding to their respective enzymes, we, the authors of this review, believe that it is imperative to discuss a few of the reported compounds.

Aryloxyphenoxypropionate herbicides (fops) such as clodinafop (Fig. 4) and cyclohexanediones (dims) have been reported to inhibit the rate-limiting enzyme of the pathway (ACCases) with $\mathrm{IC}_{50}$ values of 100-200 Mm [56]. It has been shown that these herbicides particularly inhibit carboxyltransferase domain of the apicoplastic form of ACCase, whereas the cytosolic form of this enzyme is not affected [57]. Despite the fact that these compounds have been reported to inhibit ACCase of P. falciparum with<smiles>C=C/C(C)=C/[C@@]1(C)SC(=O)C(C)=C1O</smiles>

Thiolactomycin<smiles>O=C(CC(=O)C(F)(F)F)c1ccc([N+](=O)[O-])cc1</smiles>

NAS-21<smiles>C[C@H](Oc1ccc(Oc2ncc(Cl)cc2F)cc1)C(=O)O</smiles>

Fig. 4 Different inhibitors of fatty acid synthesis. 
a concentration $>100 \mu \mathrm{M}$ [58], and that there might be safety-related issues, they still have the potential to serve as a good lead. KAS III ( $\beta$-ketoacyl-ACP synthase III) catalyzed the second step of chain initiation in this pathway (decarboxylative condensation of malonyl-ACP and acetyl-CoA, forming a $\beta$-ketoacetyl-ACP product) and the natural products ceruline ${ }^{[58]}$ and thiolactomycin (Fig. 4) [59] are well characterized inhibitors of this enzyme. In another study Lee et al. (2009) [60] have shown that $60 \%$ of submicromolar inhibitors of KAS III among sulfides, sulfonamides and sulfonyl groups screened by using pharmacophore of PfKASIII, inhibited the in vitro growth of malaria parasites. Even if there are structural differences between KAS III and KAS I/II (elongate $\beta$-acyl-ACP through condensation reaction), these enzymes share some of the inhibitors like plastencin [61]. Moreover, it was also found out that some derivatives of thiolactomycine and 1, 2-dithiole-3-one (known KAS I/II inhibitors) block KAS III with $\mathrm{IC}_{50}$ values of $5-10 \mu \mathrm{M}$ and $0.53-10.4$ $\mu \mathrm{M}$, respectively [49].

The other lead compounds, NAS-21 and NAS-91 (Fig. 4), were brought to light as inhibitors of PfHAD with $\mathrm{IC}_{50}$ values of $100 \mu \mathrm{M}$ and $7.4 \mu \mathrm{M}$, respectively [62]. Based on the homology model of PfHAD, docking studies were carried out on these compounds. The results adumbrated that both these compounds are competitive for crotonoyl-CoA and $\beta$-hydroxybutyryl-CoA and they inhibited FAS in cell-free extracts of malaria parasites [18]. Another enzyme of FAS II is Fab I. This enzyme is inhibited by the linear sesquiterpene lactones; anthecotulide, 4-hydroxyanthecotulide and 4-acetoxyanthecotulide [63]. From these compounds, the oxygenated derivatives, 4-hydroxyanthecotulide and 4-acetoxyanthecotulide, were found to inhibit both Fab G and Fab I with an $\mathrm{IC}_{50}$ value of $20-75 \mu \mathrm{g} / \mathrm{mL}$. The extract of Turkish freshwater macrophytes, Cladophroa glomerata and Ulva lactuca also showed antiplasmodial activity by inhibiting Fab I with an
$\mathrm{IC}_{50}$ of $1-4 \mu \mathrm{g} / \mathrm{ml}$ [64]. The interesting feature of these macrophytes that even makes them a good lead compound is that, they are not cytotoxic to human L6 cell line. Acetylenic fatty acids, 2-, 5-, 6-, and 9-HADs (hexadecynoic acids), were also evaluated for the inhibition of the growth of blood-stage $P$. falciparum and liver stage P. yoelii [65]. Among these compounds, 2-HAD was the only one that arrested the liver stage growth of $P$. yoelii, and also exhibited the best inhibitory activity against Fab I and Fab Z with $\mathrm{IC}_{50}$ values of 0.38 and $0.58 \mu \mathrm{g} / \mathrm{mL}$, respectively.

\section{Heme synthesis pathway}

Besides detoxifying heme that comes from host heamoglobin degradation, $P$. falciparum also synthesizes its own heme [66]. Heme is an important prosthetic group in many proteins such as cytochromes [67]. It has been demonstrated that heme biosynthetic pathway in $P$. falciparum is shared among apicoplast, mitochondrion and cytosol of the parasite [68]. From a target discovery point of view, the idea to discover the localization of different enzymes which participate in this pathway is indispensable. Moreover, because there is an overlap between mammalian and parasitic enzymes, those of endosymbiotic origin are thought to be expedient to target [16].

Heme synthesis pathway starts in mitochondria of malaria parasite with the synthesis of $\delta$-ALA (aminolaevulinic acid) from glycine and succinyl-CoA that is catalyzed by ALA synthase [69]. It has been reported that, iron chelators in addition to their ability to form toxic complex with iron, can also withhold iron from heme synthesis and cause a down-regulation in the synthesis of ALA synthase [70]. Iron chelators like deferiprone and desferrioxamine, known for their unsuitable adsorption and pharmacokinetic properties, were tried as adjunctive treatments in different controlled trials and showed positive results [71]. But considering the fact that the data from these studies were not statistically significant, these drugs are 
expensive and they have to be administered parentrally, they do not seem to be the ideal drugs to be used in malarious areas. On the other hand, FBSO701, an oral iron chelator with good pharmacokinetic properties, showed a better potency $\left(\mathrm{IC}_{50}=5 \mu \mathrm{M}\right)$ as compared to deferiprone $\left(\mathrm{IC}_{50}=15\right.$ $\mu \mathrm{M})$ and deferoxamine $\left(\mathrm{IC}_{50}=30 \mu \mathrm{M}\right)$ [72]. In this study, in addition to in vitro activity on $P$. falciparum culture in sybrgreen drug inhibition assays, a single oral dose of this compound also cured the lethal $P$. yoelii infection in mice. FBSO701 is now in Phase II human trials for the treatment of transfusional iron overload. Although, apparently, this compound is a potential candidate for combination therapy with other antimalarial compounds, no reports are available yet regarding its use in combination therapy.

After the synthesis of ALA in mitochondrion, heme biosynthetic pathway relocates itself to the apicoplast which contains enzymes like, $\delta$-aminolaevulinate dehydratase (Hem B), porphobilinogen deaminase (Hem C) and uroporphyrinogen III decarboxylase (Hem E) (Fig. 5) [68]. An interesting feature about Hem $\mathrm{B}$ is that in addition to its de novo synthesis, the parasite imports this enzyme from the host into the cytosol during its intraerythrocytic stage (Bonday et al., 2000) [73]. This feature is presumed to take about $75 \%-90 \%$ of total ALAD activity in the parasite $[74,75]$, and is considered as one of the attractive chemotherapeutic targets in the pathway. Even if there is a possibility that the parasite may backup, the reduced

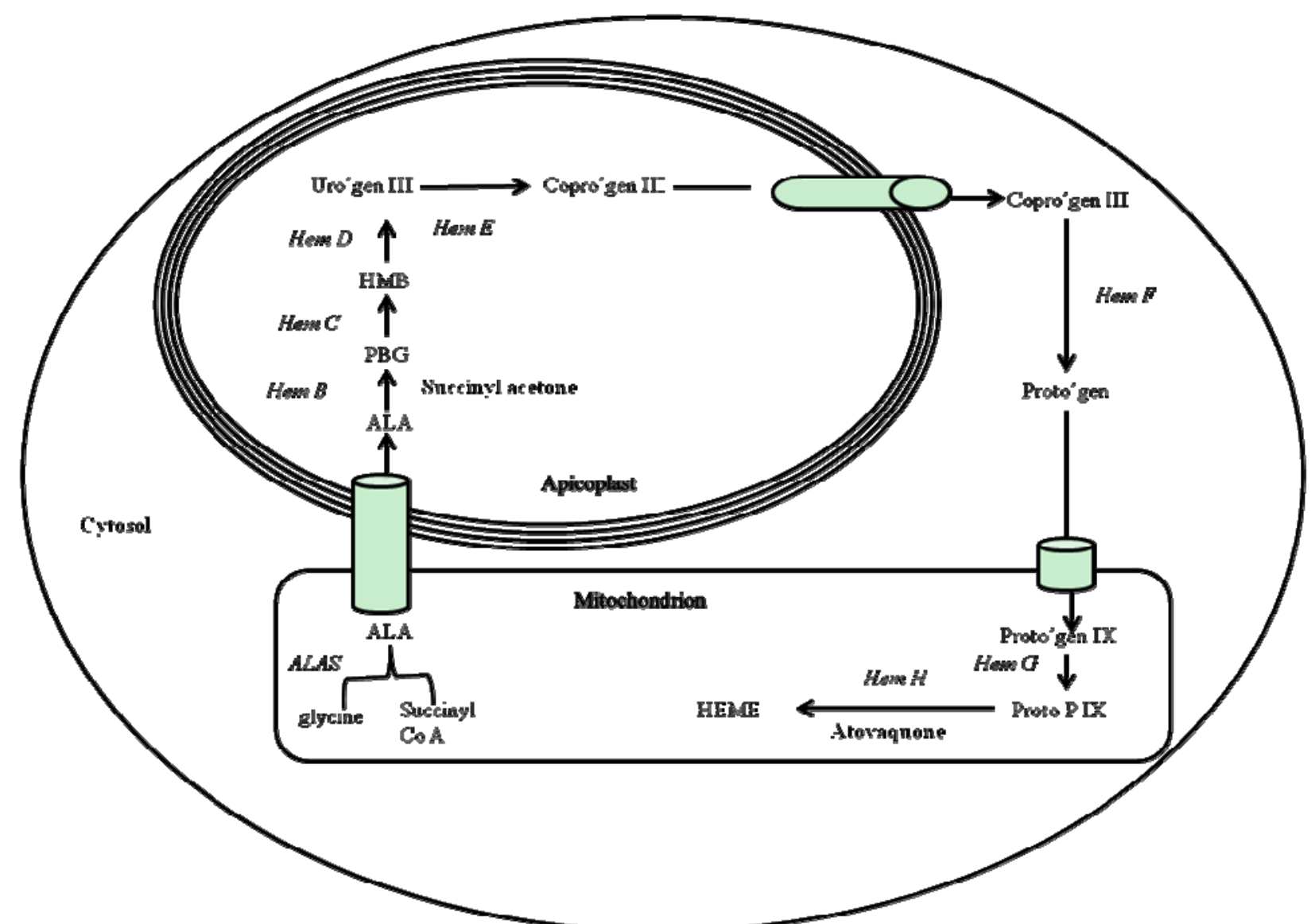

Fig. 5 Heme biosynthesis pathway in $P$. falciparum. Succinyl acetone and atovaquone inhibits Hem $B$ and Hem $H$ respectively. ALA, $\delta$ - aminolevulinic acid; ALAS, ALA synthase; HemB, porphobilinogen synthase; PBG, porphobilinogen; HemC, porphobilinogen deaminase; HMB, hydroxymethylbilane; HemD, uroporphyrinogen III synthase; UROGEN III, uroporphyrinogen III; HemE, uroporphyrinogen III decarboxylase; COPROGEN, coproporphorinogen III; HemF, coproporphorinogen oxidase; PROTOGEN IX, protoporphyrinogen IX; HemG, protoporphyrinogen oxidase; ProtoP IX, protoporphyrinogen protein $\mathrm{X}$; HemH, ferrochelatase. 
<smiles>CC(=O)CC(=O)CCC(=O)O</smiles>

Succinyl acetone<smiles>O=C1C(O)=C(C2CCC(c3ccc(Cl)cc3)CC2)C(=O)c2ccccc21</smiles>

Atovaquone<smiles>Nc1c([N+](=O)[O-])ccc(Oc2ccccc2)c1Cl</smiles>

Aclonifen<smiles>CCOc1cc(Oc2ccc(C(F)(F)F)cc2Cl)ccc1[N+](=O)[O-]</smiles>

Oxyfluorfen

Fig. 6 Inhibitors of heme synthesis.

catalytic efficiencies of its heme biosynthetic enzymes during intraerythrocytic stage by importing enzymes from the host [68], it is still controversial to the established fact that liver-stage schizogony needs more metabolic substrates than that of blood stage [76]. It has also been reported that succinyl acetone has the ability to inhibit both parasite and host ALAD, and can kill the parasite when added to the culture [77]. In addition to succinyl acetone, it has been described that $\mathrm{N}$ - and $\mathrm{C}$-terminal deleted fragment of host ALAD can compete with the enzyme for binding to $P$. berghei membrane [73]. Thus, ALAD- NC was found to decrease the enzyme's activity and heme synthesis when added to a culture of $P$. falciparum, and ultimately led to the death of the parasite.

Recently, the localization of coproporphyrinogen oxidase (Hem F) and protoporphorinogen oxidase IX (Hem G), which, for some time remained a mystery in this pathway, has been discovered by enzyme bioassay and immunoflorecence microscopy [78, 79]. The results of these studies have proved Hem $F$ is localized in cytosol and Hem G is localized in mitochondrion. Evaluation of herbicides like oxadiazon, aclonifen and oxyfluorfen (Fig. 6) against this enzyme showed the inhibition of in vitro growth of P. falciparum. ${ }^{[16]}$ In addition to the reported herbicides, atovaquone (Fig. 6), a well known inhibitor of electron transport chain (ETC)-dependent enzyme dihydroorotate dehydrogenase, was tested against this enzyme. The results showed that it could inhibit the growth of P. falciparum in culture [78]. The combination of atovaqone with proguanil (malarone) is currently in use in some parts of the world to reverse resistance against proguanil. However, the mechanism of synergism is not by inhibition of heme biosynthesis enzymes, rather it is due to the inhibition of primidine synthesis which is coupled to electron transport system [80].

Ferrochelatase (Hem H) is the last enzyme in the heme biosynthetic pathway which is localized in mitochondrion. It is responsible for insertion of ferrous iron into protoporpherin IX [81]. Heme $\mathrm{H}$ is also imported from the host to the cytosol of the parasite in a bigger proportion as compared to its de novo synthesis in the parasite. As per our literature survey, apparently, no inhibitor of $\mathrm{Hem} \mathrm{H}$, has been reported so far. Further, there are no published reports regarding the combination of the above or other compounds, which target different enzymes in the heme biosynthetic pathway. This literature position thus suggests the need to test the above mentioned compounds or their analogs in combination, to inhibit different enzymes in heme synthesis pathway, which ultimately might lead to the discovery of novel 
antimalarial drugs.

\section{Future Prospects}

Combination therapy, the current choice to retard and roll-back the resistance crisis, is probably the only option available to extend the working life of drugs, which are on the front-line in the battle against malaria. Apart from delaying the emergence of resistance, it's a bare fact that a combination of two drugs, which act on different enzymes (targets) in the same metabolic pathway, may also give good efficacy and toxicological profiles [82, 2]. So far, the search for the targets to discover new antimalarial drug has been made by the identification of new potential targets in apicoplastic metabolic pathways, which are known to be evolutionarily distant from those of the humans. Following this further, more than a few known and new lead compounds have been screened for their inhibitory activity against metabolic enzymes like DXR, DXS and FPPS in isoprenoid pathway; ENR, the KAS family, KAR and HAD in FAS II; and ALAD and PPO in heme synthesis pathways. However, after the sulphadoxine-pyrimethamine (inhibitor of dihydropteroate synthetase and dihydrofolate reductase, respectively, in folate pathway) combination, combination approaches which target different metabolic enzymes in the same pathway have apparently not been tried against malaria parasite. Thus, taking in to consideration the recent advances in the in vitro antimalarial screening assays using whole parasites, we do hope that there remains much to be done in the pursuit of new combinations of drugs which target different enzymes belonging to one particular metabolic pathway.

\section{Acknowledgments}

We are grateful to Prof. K. K. Bhutani, Officiating Director, National Institute of Pharmaceutical Education and Research (NIPER), for his help and encouragement. S. T. thanks Government of the Federal Democratic Republic of Ethiopia for financial support. B.P. is grateful to NIPER for financial assistance. This is NIPER communication No. 496.

\section{References}

[1] Fact Sheet World Malaria Report. 2014. http://www.who.int/malaria/media/world_malaria_report 2014/en/.

[2] Snow, R. W., Guerra, C. A., Noor, A. M., Myint, H. Y., and Hay, S. I. 2005. "The Global Distribution of Clinical Episodes of Plasmodium falciparum Malaria." Nature 434 (7030): 214-17.

[3] Goselle, O. N., Onwuliri, C. O., and Onwuliri, V. A. 2009. "Malaria Infection in HIV/AIDS Patients and its Correlation with Packed Cell Volume (PCV)." Journal of Vector Borne Diseases 46 (3): 205-11.

[4] Skinner-Adams, T. S., McCarthy, J. S., Gardiner, D. L., and Andrews, K. T. 2008. "HIV and Malaria Co-infection: Interactions and Consequences of Chemotherapy." Trends in Parasitology 24 (6): 264-71.

[5] Cibulskis, R. E., Bell, D., Christophel, E. M., Hii, J., Delacollette, C., Bakyatia, N., and Aregawi, M. W. 2007. "Estimating Trends in the Burden of Malaria at Country Level." American Journal of Tropical Medicine and Hygiene 77 (6): 133-37.

[6] Davis T. M., Karunajeewa, H. A., and Llett, K. F. 2005. "Artemisinin-Based Combination Therapies for Uncomplicated Malaria." Medical Journal of Australia 182 (4): 181-85.

[7] Dondorp, A. M., Nosten, F., Yi, P., Das, D., Phyo, A. P.; Tarning, J., Lwin, K. M., Ariey, F., Hanpithakpong, W., Lee, S. J., Ringwald, P., Silamut, K., Imwong, M., Chotivanich, K., Lim, P., Herdman, T., An, S. S., Yeung, S., Singhasivanon, P., Day, NP., Lindegardh, N., Socheat, D., and White, N. J. 2009. "Artemisinin Resistance in Plasmodium falciparum Malaria." New England Journal of Medicine 36: 455-67.

[8] Na-Bangchang, K., Ruengweerayut, R., Mahamad P., Ruengweerayut, K., and Chaijaroenkul, W. "Declining in Efficacy of a Three-day Combination Regimen of Mefloquine-Artesunate in a Multi-drug Resistance Area along the Thai-Myanmar Border." Malaria Journal 9: 273-77.

[9] Tun, K. M., Imwong, M., Lwin, K. M., Win, A. A., Hlaing, T. M., Hlaing, T., Lin, K., Kyaw, M. P., Plewes, K., Faiz, M. A., Dhorda, M., Cheah, P. Y., Pukrittayakamee, S., Ashley E. A., Anderson, T. J., Nair, S., McDew-White, M., Flegg, J. A., Grist, E. P., Guerin, P., Maude, R. J., Smithuis, F., Dondorp, A. M., Day, N. P., Nosten, F.,White, N. J., and Woodrow, C. J. 2015. "Spread of Artemisinin-Resistant Plasmodium falciparum in Myanmar: A Cross-Sectional Survey of the K13 
Molecular Marker." Lancet Infectious Diseases 15 (4): 415-21.

[10] Chawira, A. N., Warhurst, D. C., Robinson, B. L., and Peters, W. 1987. "The Effect of Combinations of Qinghaosu (artemisinin) with Standard Antimalarial Drugs in the Suppressive Treatment of Malaria in Mice." Transactions of Royal Society of Tropical Medicine and Hygiene 81(4): 554-58.

[11] Janouskovec, J., Horák, A., Oborník, M., Lukes, J., and Keeling, P. J. 2010. "A Common Red Algal Origin of the Apicomplexan, Dinoflagellate, and Heterokont Plastids." Proceedings of the National Academy of. Sciences of the United States of America 107 (24): 10949-54.

[12] Seeber, F. 2003. "Biosynthetic Pathways of Plastid-Derived Organelles as Potential Drug Targets against Parasitic Apicomplexa." Current Drug Targets-Immune, Endocrine \&Metabolic Disorders 3 (2): 99-09.

[13] Seeber, F., and Soldati-Favre, D. 2010. "Metabolic Pathways in the Apicoplast of Apicomplexa." International Review of Cell and Molecular Biology 281: 162-28.

[14] Botté, C. Y., Dubar, F., McFadden, G. I., Maréchal, E., and Biot, C. 2012. "Plasmodium falciparum Apicoplast Drugs: Targets or Off-Targets?" Chemical Reviews 112 (3): 1269-83.

[15] Ramya, T. N., Mishra, S., Karmodiya, K., Surolia, N., and Surolia, A. 2007. "Inhibitors of Nonhousekeeping Functions of the Apicoplast Defy Delayed Death in Plasmodium falciparum." Antimicrobial Agents and Chemotherapy 51 (1): 307-16.

[16] Wiesner, J., and Seeber, F. 2005. "The Plastid-Derived Organelle of Protozoan Human Parasites as a Target of Established and Emerging Drugs." Expert Opinion on Therapeutic Targets 9 (1): 23-44.

[17] McFadden, G. I., and Roos, D. S. 1999. "Apicomplexan Plastids as Drug Targets." Trends in Microbiology 7 (8): 328-33.

[18] Surolia, A., Ramya, T. N., Ramya, V., and Surolia, N. 2004. "FAS't Inhibition of Malaria." Biochemical Journal 383 (3): 401-12.

[19] Surolia, N., and Surolia, A. 2001. "Triclosan Offers Protection against Blood Stages of Malaria by Inhibiting Enoyl-ACP Reductase of Plasmodium falciparum." Nature Medicine 7 (2): 167-73.

[20] Jomaa, H., Wiesner, J., Sanderbrand, S., Altincicek, B., Weidemeyer, C., Hintz, M., Türbachova, I., Eberl, M., Zeidler, J., Lichtenthaler, H. K., Soldati, D., and Beck, E. 1999. "Inhibitors of the Nonmevalonate Pathway of Isoprenoid Biosynthesis as Antimalarial Drugs." Science 285 (5433): 1573-76.
[21] Nzila, A. 2006. "Inhibitors of de novo Folate Enzymes in Plasmodium falciparum." Drug Discovery Today 11 (19-20): 939-44.

[22] Rosenthal, P. J. 1998. "Proteases of Malaria Parasites: New Targets for Chemotherapy." Emerging Infectious Diseases 4 (1): 49-57.

[23] Goldstein, J. L., and Brown, M. S. 1990. "Regulation of the Mevalonate Pathway." Nature 343 (6257): 425-30.

[24] Barkovich, R., and Liao, J. C. 2001. "Review: Metabolic Engineering of Isoprenoids." Metabolic Engineering 3 (1): 27-39.

[25] Rohmer, M. 2003. "Mevalonate-Independent Methylerythritol Phosphate Pathway for Isoprenoid Biosynthesis Elucidation and Distribution." Pure and Applied Chemistry 75 (2-3): 375-88.

[26] Lichtenthaler, H. K., Schwender, J., Disch, A., and Rohmer, M. 1997. "Biosynthesis of Isoprenoids in Higher Plant Chloroplasts Proceeds via a Mevalonate-Independent Pathway." FEBS Letters 400 (3): 271-74.

[27] Eisenreich, W., Bacher, A., Arigoni, D., and Rohdich, F. 2004. "Biosynthesis of Isoprenoids via the Non-Mevalonate Pathway." Cellular and Molecular Life Sciences 61 (12): 1401-26.

[28] Hirsch A. H., and Diederich, F. 2008. "The Non-Mevalonate Pathway to Isoprenoid Biosynthesis: A Potential Source of New Drug Targets.” Chimia 62 (4): 226-30.

[29] Rodriguez, C. M. 2004. "The MEP Pathway: A New Target for the Development of Herbicides, Antibiotics and Antimalarial Drugs." Current Pharmaceutical Design 10 (19): 2391-00.

[30] Singh, A. P., Zhang, Y., No, J. H., Docampo, R., Nussenzweig, V., and Oldfield, E. 2010. "Lipophilic Bisphosphonates are Potent Inhibitors of Plasmodium Liver-Stage Growth." Antimicrobial Agents and Chemotherapy 54 (7): 2987-93.

[31] Kuzuyama, T., Shimizu, T., Takahashi, S., and Seto, H. 1998. "Fosmidomycin, a Specific Inhibitor of 1-Deoxy-Xylulose 5-Phosphate Reductoisomerase in the Nonmevalonate Pathway for Terpenoid Biosynthesis." Tetrahedron Letters. 39 (43): 7913-16.

[32] Grellier, P., Depoix, D., Schrével, J., and Florent, I. 2008. "Discovery of New Targets for Antimalarial Chemotherapy." Parasite 15 (3): 219-25.

[33] Zhang, B., Watts, K. M., Hodge, D., Kemp, L. M., Hunstad, D. A., Hicks, L. M., and Odom, A. R. 2011. “A Second Target of the Antimalarial and Antibacterial Agent Fosmidomycin Revealed by Cellular Metabolic Profiling." Biochemistry 50 (17): 3570-77.

[34] Mueller, C., Schwender, J., Zeidler, J., and Lichtenthaler, H. K. 2000. "Properties and Inhibition of the First Two 
Enzymes of the Non-Mevalonate Pathway of Isoprenoid Biosynthesis.” Biochemical Society Transactions 28 (6): 792-03.

[35] Altincicek, B., Hintz, M., Sanderbrand, S., Wiesner, J., Beck, E., and Jomaa, H. 2000. "Tools for Discovery of Inhibitors of the 1-Deoxy-D-Xylulose 5-Phosphate (DXP) Synthase and DXP Reductoisomerase: an Approach with Enzymes from the Pathogenic Bacterium Pseudomonas aeruginosa." FEMS Microbiology Letters 190 (2): 329-33.

[36] Wang, W., Wang, K., Liu, Y. L., No, J. H., Li, J., Nilges, M. J.,and Oldfield, E. 2010. "Bioorganometallic Mechanism of Action, and Inhibition, of IspH." Proceedings of the National Academy of. Sciences of the United States of America 107 (10): 4522-27.

[37] Zhang, Y., Cao, R., Yin, F., Hudock, M. P., Guo, R. T., Krysiak, K., Mukherjee, S., Gao, Y. G., Robinson, H., Song, Y., No, J. H., Bergan, K., Leon, A., Cass, L., Goddard, A., Chang, T. K., Lin, F. Y., Van Beek, E., Papapoulos, S., Wang, A. H., Kubo, T., Ochi, M., Mukkamala, D., and Oldfield, E. 2009. "Lipophilic Bisphosphonates as Dual Farnesyl/Geranylgeranyl Diphosphate Synthase Inhibitors: an X-Ray and NMR Investigation." Journal of American Chemical Society 131 (14): 5153-62.

[38] Jordão, F. M., Saito, A. Y., Miguel, D.C., de Jesus Peres, V., Kimura, E. A., and Katzin, A. M. 2011. "In-vitro and In-vivo Antiplasmodial Activity of Risedronate and its Interference with Protein Prenylation in P. falciparum." Antimicrobial Agents and Chemotherapy 55 (5): 2026-31.

[39] Kunzmann, V., Bauer, E., and Wilhelm, M. 1999. "Gamma/delta T-cell Stimulation by Pamidronate." New England Journal of Medicine 340 (9): 737-38.

[40] Mönkkönen, H., Auriola, S., Lehenkari, P., Kellinsalmi, M., Hassinen, I. E., Vepsäläinen, J., and Mönkkönen J. 2006. “A New Endogenous ATP Analog (ApppI) Inhibits the Mitochondrial Adenine Nucleotide Translocase (ANT) and is Responsible for the Apoptosis Induced by Nitrogen Containing Bisphosphonates." British Journal of Pharmacology 147 (4): 437-45.

[41] Leon, A., Liu, L., Yang, Y., Hudock, M. P., Hall, P., Yin, F., Studer, D., Puan, K. J., Morita, C. T., and Oldfield, E. 2006. "Isoprenoid Biosynthesis as a Drug Target: Bisphosphonate Inhibition of Escherichia Coli K12 Growth and Synergistic Effects of Fosmidomycin." Journal of Medicinal Chemistry 49 (25): 7331-41.

[42] Mazumdar, J., and Striepen, B. 2007. "Make It or Take It: Fatty Acid Metabolism of Apicomplexan Parasites." Eukaryotic Cell 6 (10): 1727-35.

[43] Ralph, S. A., van Dooren, G. G., Waller, R. F., Crawford, M. J., Fraunholz, M. J., Foth, B. J., Tonkin, C. J., Roos, D. S., and McFadden, G. I. 2004. "Tropical
Infectious Diseases: Metabolic Maps and Functions of the Plasmodium falciparum Apicoplast." Nature Reviews Microbiology 2 (3): 203-16.

[44] Waller, R. F., Reed, M. B., Cowman, A. F., and McFadden, G. I. 2000. "Protein Trafficking to the Plastid of Plasmodium falciparum is via the Secretory Pathway." EMBO Journal 19 (8): 1794-02.

[45] Toler, S. 2005. "The Plasmodial Apicoplast Was Retained Under Evolutionary Selective Pressure to Assuage Blood Stage Oxidative Stress." Medical Hypotheses 65 (4): 683-90.

[46] Foth, B. J., and McFadden, G. I. 2003. "The Apicoplast: A Plastid in Plasmodium falciparum and other Apicomplexan Parasites." International Review of Cytology 224: 57-10.

[47] Waters, N. C., Kopydlowski, K. M., Guszczynski, T., Wei, L., Sellers, P., Ferlan, J. T., Lee, P. J., Li, Z., Woodard, C. L., Shallom, S., Gardner, M. J., and Prigge, S. T. 2002. "Functional Characterization of the Acyl Carrier Protein (Pfacp) and Beta-Ketoacyl ACP Synthase III (Pfkasiii) from Plasmodium falciparum.” Molecular and Biochemical Parasitology. 123 (2): 85-94.

[48] Pillai, S., Rajagopal, C., Kapoor, M., Kumar, G., Gupta, A., and Surolia, N. 2003. "Functional Characterization of [Beta]-Ketoacyl-ACP Reductase (FabG) from Plasmodium falciparum." Biochemical and Biophysical Research Communications 303 (1): 387-92.

[49] Gornicki, P. 2003. "Apicoplast Fatty Acid Biosynthesis as a Target for Medical Intervention in Apicomplexan Parasites.” International Journal for Parasitology 33 (9): 885-96.

[50] Sato, S., and Wilson, J. M. 2005. "The Plastid of Plasmodium Spp.: A Target for Inhibitors." Current Topics in Microbiology and Immunology 295: 251-73.

[51] Yu, M., Kumar, T. R., Nkrumah, L. J., Coppi, A., Retzlaff, S., Li, C. D., Kelly, B. J., Moura, P. A., Lakshmanan, V., Freundlich, J. S., Valderramos, J. C., Vilcheze, C., Siedner, M., Tsai, J. H., Falkard, B., Sidhu, A. B., Purcell, L. A., Gratraud, P., Kremer, L., Waters, A. P., Schiehser, G., Jacobus, D. P., Janse, C. J., Ager, A., Jacobs, W. R., Jr Sacchettini, J. C., Heussler, V., Sinnis, P., and Fidock, D. A. 2008. "The Fatty Acid Biosynthesis Enzyme Fab I Plays A Key Role in the Development of Liver-Stage Malarial Parasites." Cell Host \& Microbe 4 (6): 567-78.

[52] Vaughan, A. M., O'Neill, M. T., Tarun, A. S., Camargo, N., Phuong, T. M., Aly, A. S., Cowman, A. F., and Kappe, S. H. 2009. "Type II Fatty Acid Synthesis is Essential only for Malaria Parasite Late Liver Stage Development." Cellular Microbiology 11 (3): 506-20.

[53] Wickramasinghe, SR., Inglis, K. A., Urch, J. E., Müller, S., van Aalten, D. M., and Fairlamb, A. H. 2006. "Kinetic, 
Inhibition and Structural Studies On 3-Oxoacyl-ACP Reductase from Plasmodium falciparum, a Key Enzyme in Fatty Acid Biosynthesis." Biochemical Journal 393 (2): 447-57.

[54] Tarun, A. S., Peng, X., Dumpit, R. F., Ogata, Y., Silva-Rivera, H., Camargo, N., Daly, T. M., Bergman, L. W., and Kappe, S. H. 2008. "A Combined Transcriptome and Proteome Survey of Malaria Parasite Liver Stages." Proceedings of the National Academy of Sciences of the United States of America 105 (1): 305-10.

[55] Daily, J. P., Scanfeld, D., Pochet, N., Le Roch, K., Plouffe, D., Kamal, M., Sarr, O., Mboup, S., Ndir, O., Wypij, D., Levasseur, K., Thomas, E., Tamayo, P., Dong, C., Zhou, Y., Lander, E. S., Ndiaye, D., Wirth, D., Winzeler, E. A., Mesirov, J. P., and Regev, A. 2007. "Distinct Physiological States of Plasmodium falciparum in Malaria-Infected Patients." Nature 450 (7172): 1091-95.

[56] Lu, J. Z., Lee, P. J., Waters, N. C., and Prigge, S. T. 2005. "Fatty Acid Synthesis as a Target for Antimalarial Drug Discovery." Combinatorial Chemistry and High Throughput Screening 8 (1): 15-26.

[57] Jelenska, J., Sirikhachornkit, A., Haselkorn, R., Gornicki, P. 2002. "The Carboxyltransferase Activity of the Apicoplast Acetyl-CoA Carboxylase of Toxoplasma Gondii is the Target of Aryloxyphenoxypropionate Inhibitors." Journal of Biological Chemistry 277 (26): 23208-15.

[58] Waller, R. F., Ralph, S. A., Reed, M. B., Su, V., Douglas, J. D., Minnikin, D. E., Cowman, A. F., Besra, G. S., and McFadden, G. I. 2003. "A Type II Pathway for Fatty Acid Biosynthesis Presents Drug Targets in Plasmodium falciparum." Antimicrobial Agents and Chemotherapy 47 (1): 297-01.

[59] Jones, S. M., Urch, J. E., Brun, R., Harwood, J. L., Berry, C., and Gilbert I. H. 2004. "Analogues of Thiolactomycin as Potential Anti-Malarial and Anti-Trypanosomal Agents." Bioorganic and Medicinal Chemistry 12 (4): 683-92.

[60] Lee, P. J., Bhonsle, J. B., Gaona, H. W., Huddler, D. P., Heady, T. N., Kreishman-Deitrick, M., Bhattacharjee, A., McCalmont, W. F., Gerena, L., Lopez-Sanchez, M., Roncal, N. E., Hudson, T. H., Johnson, J. D., Prigge, S. T., and Waters, N. C. 2009. "Targeting the Fatty Acid Biosynthesis Enzyme, -Ketoacyl- Acyl Carrier Protein Synthase III (PfKASIII), in the Identification of Novel Antimalarial Agents." Journal of Medicinal Chemistry 52 (4): 952-63.

[61] Goodman, C. D., and McFadden, G. I. 2008. "Fatty Acid Synthesis in Protozoan Parasites: Unusual Pathways and Novel Drug Targets." Current Pharmaceutical Design 14 (9): 901-16.
[62] Sharma, S. K., Kapoor, M., Ramya, T. N., Kumar, S., Kumar, G., Modak, R., Sharma, S., Surolia, N., and Surolia, A. 2003. "Identification, Characterization, And Inhibition Of Plasmodium falciparum -Hydroxyacyl-Acyl Carrier Protein Dehydratase (FabZ)." Journal of Biological Chemistry 278 (46): 45661-71.

[63] Karioti, A., Skaltsa, H., Zhang, X., Tonge, P. J., Perozzo, R., Kaiser, M., Franzblau, S. G., and Tasdemir, D. 2008. "Inhibiting Enoyl-ACP Reductase (FabI) Across Pathogenic Microorganisms by Linear Sesquiterpene Lactones from Anthemis auriculata." Phytomedicine 15 (12): 1125-29.

[64] Orhan, I., Sener, B., Atici, T., Brun, R., Perozzo, R., and Tasdemir, D. 2006. "Turkish Freshwater and Marine Macrophyte Extracts Show In-vitro Antiprotozoal Activity and Inhibit FabI, A Key Enzyme of Plasmodium falciparum Fatty Acid Biosynthesis." Phytomedicine 13 (6): 388-93.

[65] Tasdemir, D, Sanabria, D., Lauinger, I. L., Tarun, A., Herman, R., Perozzo, R., Zloh, M., Kappe, S. H., Brun, R., and Carballeira, N. M. 2010. "2-Hexadecynoic Acid Inhibits Plasmodial FAS-II Enzymes and Arrest Erythrocytic and Liver Stage Plasmodium Infections." Bioorganic and Medicinal Chemistry 18 (21): 7475-85.

[66] Bonday, Z. Q., Taketani, S., Gupta, P. D., and Padmanaban, G. 1997. "Heme Biosynthesis by the Malarial Parasite." Journal of Biological Chemistry 272: 21839-46.

[67] Ferreira, A., Balla, J., Jeney, V., Balla, G, Soares, M. P. 2008. "A Central Role for Free Heme in the Pathogenesis of Severe Malaria: The Missing Link?" Journal of Molecular Medicine 86 (10): 1097-11.

[68] Lim, L., and McFadden, G. I. 2010. "The Evolution, Metabolism and Functions of the Apicoplast." Philsophical Transactions of the Royal Society of London Series B Biological Sciences 365: 749-63.

[69] Varadharajan, S., Dhanasekaran, S., Bonday, Z. Q., Rangarajan, P. N., and Padmanaban, G. 2002. "Involvement of Delta-Aminolaevulinate Synthase Encoded by the Parasite Gene in de novo Haem Synthesis By Plasmodium falciparum." Biochemical Journal 367 (2): 321-27.

[70] Mabeza, G. F., Biemba, G., and Gordeuk, V. R. 1996. "Clinical Studies of Iron Chelators in Malaria." Acta Haematologica 95 (1): 78-86.

[71] Smith, H. J., and Meremikwu, M. M. 2003. "Iron-Chelating Agents for Treating Malaria." Cochrane Database Systemic Reviews 10.1002/14651858.CD001474.

[72] Tripathi, A., Rienhoff, H., and Sullivan, D. 2010. "Single Oral Dose Cure of Lethal $P$. yoelii with a New Iron Chelator in Human Clinical Trials for Iron Overload." 
Malaria Journal 9 (2): 01-02.

[73] Bonday, Z. Q., Dhanasekaran, S., Rangarajan, P. N., and Padmanaban, G. 2000. "Import of Host -Aminolevulinate Dehydratase in to the Malarial Parasite: Identification of a New Drug Target." Nature Medicine 6 (8): 898-03.

[74] Dhanasekaran, S., Chandra, N. R., Sagar, B. K., Rangarajan, P. N., and Padmanaban G. 2003."D-Aminolevulinate Dehydrase from Plasmodium falciparum-Indigenous vs Imported." Journal of Biological Chemistry 279: 6934-42.

[75] Padmanaban, G., Nagaraj,V. A., and Rangarajan, P. N. 2007. "An Alternative Model for Heme Biosynthesis in the Malarial Parasite." Trends in Biochemical Sciences 32 (10): 443-49.

[76] Prudêncio, M., Rodriguez, A., and Mota, M. M. 2006. "The Silent Path to Thousands of Merozoites: The Plasmodium Liver Stage." Nature Reviews Microbiology 4 (11): 849-56.

[77] Surolia, N., and Padmanaban, G. 1992. "Biosynthesis of Heme Offers a New Chemotherapeutic Target in the Human Malarial Parasite." Biochemical and Biophysical
Research Communications 187 (2): 744-750.

[78] Nagaraj, V. A., Arumugam, R., Prasad, D., Rangarajan, P. N, and Padmanaban, G. 2010a. "Protoporphyrinogen IX Oxidase from Plasmodium falciparum is Anaerobic and is Localized to the Mitochondrion." Molecular and Biochemical Parasitology 174 (1): 44-52.

[79] Nagaraj, V. A., Prasad, D., Arumugam, R., Rangarajan, P. N., and Padmanaban G. 2010b. "Characterization of Coproporphyrinogen III Oxidase in Plasmodium falciparum Cytosol.” Parasitology International 59 (2): 121-27.

[80] McBride, J. H. 2010. "Chemoprophylaxis of Tropical Infectious Diseases." Pharmaceuticals 3: 1561-75.

[81] van Dooren, G. G., Stimmler, L. M., and McFadden, G. I. 2006. "Metabolic Maps and Functions of the Plasmodium Mitochondrion." FEMS Microbiology Reviews 30 (4): 596-30.

[82] Olliaro, P. L., and Taylor, W. R. 2004. "Developing Artemisinin Based Drug Combinations for the Treatment of Drug Resistant Falciparum Malaria: A Review." Journal of Postgraduate Medicine 50 (1): 40-44. 